\title{
T-helper cell type 2 (Th2) memory T cell-potentiating cytokine IL-25 has the potential to promote angiogenesis in asthma
}

\author{
Chris J. Corrigan ${ }^{\mathrm{a}, 1}$, Wei Wang, ${ }^{\mathrm{b}, c, 1}$, Qiu Meng ${ }^{\mathrm{a}}$, Cailong Fang ${ }^{\mathrm{a}}$, Huifen Wu ${ }^{\mathrm{a}}$, Victoria Reay ${ }^{\mathrm{a}}$, Ze Lv ${ }^{\mathrm{b}, \mathrm{c}}$, Yiqiang Fan ${ }^{\mathrm{b}, \mathrm{c}}$, \\ Yunqing An ${ }^{b, c, 2}$, Yui-Hsi Wang ${ }^{d, e, f}$, Yong-Jun Liu, ${ }^{\text {e,f }}$, Tak H. Lee ${ }^{a}$, and Sun Ying ${ }^{a, 2}$ \\ aDivision of Asthma, Allergy and Lung Biology, King's College London, Medical Research Council, and Asthma UK Centre in Allergic Mechanisms of Asthma, \\ London SE1 9RT, United Kingdom; Departments of 'Immunology and 'Respiratory Diseases, Capital Medical University, Beijing 100069, People's Republic of

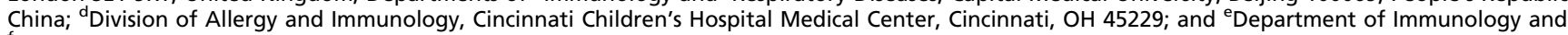 \\ ${ }^{f}$ Centre of Cancer Immunology Research, University of Texas M. D. Anderson Cancer Center, Houston, TX 77054
}

Edited* by K. Frank Austen, Brigham and Women's Hospital, Boston, MA, and approved December 9, 2010 (received for review September 23, 2010)

IL-25 (IL-17E) is a T-helper cell type 2 (Th2) cytokine best described as a potentiator of Th2 memory responses. Reports of expression of its receptor, IL-25R, on airways structural cells suggest a wider role for IL-25 in remodeling. We hypothesized that IL-25 stimulates local angiogenesis in the asthmatic bronchial mucosa. Immunoreactive IL- $25^{+}, \mathrm{IL}-25 \mathrm{R}^{+}$, and $\mathrm{CD} 31^{+}$(endothelial) cells in sections of bronchial biopsies from asthmatics and controls were detected by immunohistochemistry. The effect of IL-25 on angiogenesis was examined using an in vitro assay. Real-time PCR was used to detect expression of IL-25R and VEGF mRNA in cultured human vascular endothelial cells (HUVEC), and a cell proliferation kit (WST-8) was used to measure the effect of IL-25 on HUVEC proliferation. Immunostaining showed that IL-25+, IL-25R ${ }^{+}$, and $\mathrm{CD} 31^{+} / \mathrm{IL}-25 \mathrm{R}^{+}$cells were significantly elevated in the bronchial mucosa of asthmatics compared with controls $(P<0.003)$. In asthmatics, the numbers of IL- $25^{+}$cells correlated inversely with the forced expiratory volume in $1 \mathrm{~s}(r=-0.639 ; P=0.01)$. In vitro, HUVEC constitutively expressed IL-25R, which was up-regulated further by TNF- $\alpha$. IL-25 and TNF- $\alpha$ also increased expression of VEGF and VEGF receptors. IL-25 increased HUVEC proliferation and the number, length, and area of microvessel structures in a concentration-dependent manner in vitro. VEGF blockade, the PI3K-specific inhibitor LY294002, and the MAPK/ERK1/2 (MEK1/2)-specific inhibitor U0126 all markedly attenuated IL-25-induced angiogenesis, and the inhibitors also reduced IL-25-induced proliferation and VEGF expression. Our findings suggest that IL-25 is elevated in asthma and contributes to angiogenesis, at least partly by increasing endothelial cell VEGF/VEGF receptor expression through PI3K/Akt and Erk/MAPK pathways.

A irways remodeling in asthma refers to structural changes, which include increased angiogenesis (1-4). Previous studies suggest that neovascularization and microvascular leakage are prominent in asthmatic airways (1-5). VEGF is one of the most potent proangiogenic factors (6).

IL-25 (IL-17E) is a member of family of six proteins labeled IL-17A-F. IL-17A and IL-17F are expressed by a novel subset of $\mathrm{CD}^{+}$T-helper (Th) cells and appear to play critical roles in inflammation and autoimmunity, whereas IL-25 is exceptional in promoting Th cell type 2 (Th2) immune responses (7). In mice, exogenous administration of IL-25 $(8,9)$ or transgenic expression $(10,11)$ induces asthma-like features. Conversely, anti-IL-25 antibody reduces airways inflammation in animal models of asthma $(12,13)$. In addition to T cells, lung epithelial cells, mast cells, basophils, and eosinophils may produce IL-25 $(14,15)$.

The IL-25 receptor (IL-25R) is a 56-kDa single-transmembrane protein. We demonstrated (14) that human Th2 central memory cells selectively up-regulate IL-25R when stimulated with thymic stromal lymphopoietin-activated dendritic cells or with T-cell memory "homeostatic" cytokines such as IL-7 or when triggered by specific antigen. This up-regulation resulted in enhanced sensitivity of the cells to IL-25 that was associated with IL-4 independent, sustained expression of GATA3, c-MAF, and
JunB. This finding and the further observation (16) that IL-25 activates eosinophils to produce a range of asthma-relevant mediators suggest that IL-25 may play a pivotal role in maintaining Th2 central memory and sustaining asthmatic inflammation, whereas its production by mast cells and eosinophils suggests the possibility of a positive feedback amplifying loop.

IL-25R also is expressed on human eosinophils, monocytes, airways smooth muscle cells, and fibroblasts (16-19), raising the possibility that IL-25 also may be involved in causing structural changes in the airways that characterize asthma. However, its possible role in angiogenesis has never been explored. Our preliminary data (20) showed that immunoreactive IL-25R colocalized with $\mathrm{CD} 31^{+}$blood vessel endothelial cells. Thus, for the present study, we hypothesized that IL-25 plays a role in angiogenesis in asthma. Our aim was to measure expression of IL-25 and its receptor in the bronchial mucosa of asthmatics and controls (in controls, particularly on vascular endothelial cells) and to characterize the phenomenon and mechanisms of IL-25induced angiogenesis.

\section{Results}

Clinical Data. In this study we compared bronchial biopsies from 15 asthmatics and 15 controls. Clinical and demographic details of the study subjects are summarized in Table S1.

IL-25 and IL-25R Immunoreactive Cells and CD31 $/$ IL25R $^{+}$Microvessels in Vivo. The median number of cells showing immunoreactivity for IL-25 and IL-25R was significantly higher in the bronchial mucosa of the asthmatics than in controls (Fig. $1 A, B, E$, and $F$ ). In the asthmatics, the number of IL-25- but not IL-25Rimmunoreactive cells correlated inversely with the forced expiratory volume in $1 \mathrm{~s}(r=-0.639 ; P=0.01)$. The total number of IL-25-immunoreactive cells correlated positively with the number of IL-25R-immunoreactive cells $(r=0.522, P=0.046)$. IL-25-immunoreactive cells were located in both the epithelium and the submucosa, whereas IL-25R-immunoreactive cells were located almost exclusively in the submucosa (Fig. $1 A$ and $B$ ). The number of $\mathrm{CD} 31^{+}$and $\mathrm{CD} 31^{+} / \mathrm{IL}-25 \mathrm{R}^{+}$vascular structures

Author contributions: C.J.C., W.W., Y.A., Y.-H.W., Y.-J.L., T.H.L., and S.Y. designed research; C.J.C., W.W., Q.M., C.F., H.W., V.R., Z.L., Y.F., and S.Y. performed research; C.J. C., W.W., Q.M., C.F., H.W., Y.A., Y.-H.W., Y.-J.L., T.H.L., and S.Y. contributed new reagents/ analytic tools; C.J.C., W.W., Q.M., C.F., H.W., Y.A., Y.-J.L., T.H.L., and S.Y. analyzed data; and C.J.C., W.W., Y.A., Y.-J.L., T.H.L., S.Y. wrote the paper.

The authors declare no conflict of interest.

*This Direct Submission article had a prearranged editor.

Freely available online through the PNAS open access option.

${ }^{1}$ C.J.C. and W.W. contributed equally to this work.

${ }^{2}$ To whom correspondence may be addressed. E-mail: anyunq@ccmu.edu.cn or ying.sun@ kcl.ac.uk.

This article contains supporting information online at www.pnas.org/lookup/suppl/doi:10. 1073/pnas.1014241108/-/DCSupplemental. 

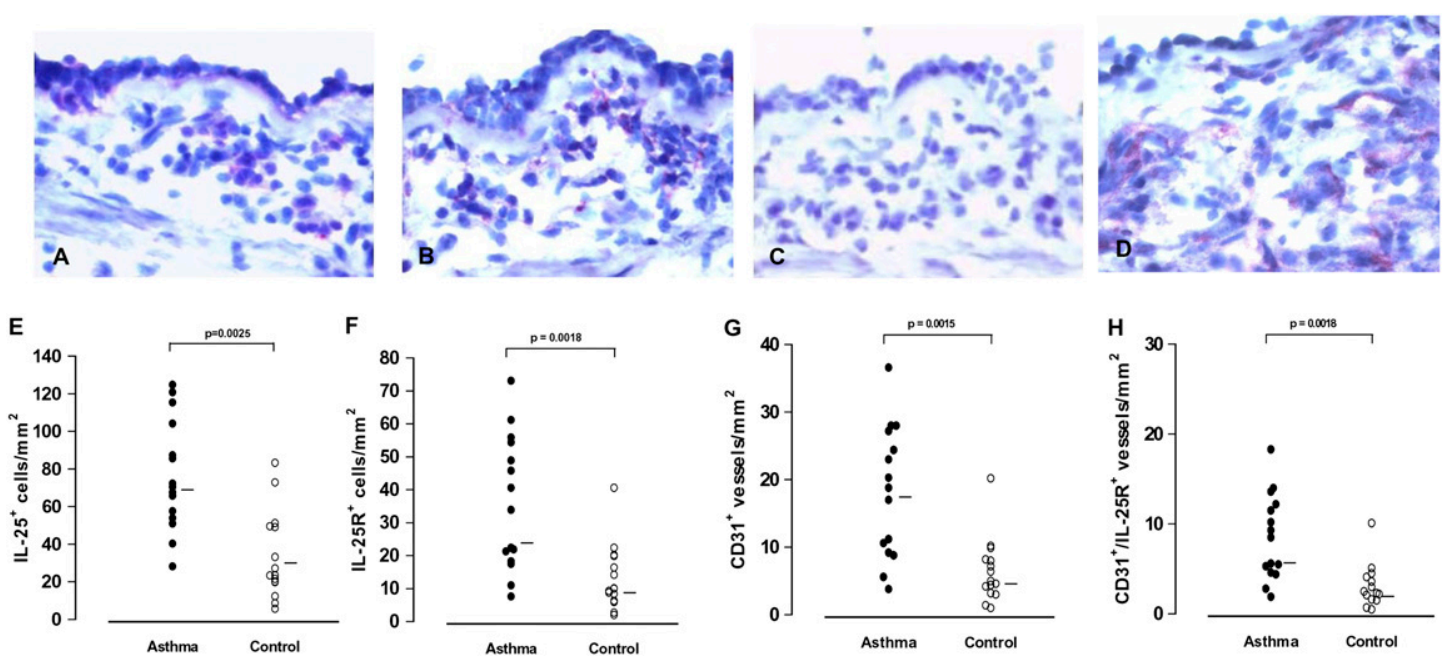

Fig. 1. (A-D) Immunoreactivity for IL-25 (A), IL-25R (B), CD31 (D), and negative control staining for IL-25 (C) in sections of the asthmatic bronchial mucosa. $(E-H)$ Total cells per unit area of entire sections showing IL-25 $(E)$ and IL-25R $(F)$ immunoreactivity and vascular structures showing CD31 $(G)$ and $C D 31^{+} / I L-25 R^{+}$ $(H)$ immunoreactivity in bronchial biopsies from asthmatics and controls ( $n=15$ for each group). Bars indicate medians. Mann-Whitney $U$ test.

also was significantly higher in asthmatics than in controls (Fig. 1 $G$ and $H$ ).

IL-25R Expression in Human Vascular Endothelial Cells in Vitro. In human vascular endothelial cells (HUVEC), constitutively expressed IL-25R mRNA, protein, and immunoreactivity were increased in the presence of TNF- $\alpha$ (Fig. $2 B, E, G$, and $H$ ). Interestingly, IL-25 also up-regulated mRNA and protein encoding its own receptor in a concentration-dependent fashion (Fig. $2 C, F, G$, and $I$ ). Western blotting showed that the expression of IL-25R protein was maximal $\approx 48 \mathrm{~h}$ after stimulation (Fig. 2G).

IL-25-Induced Angiogenesis and VEGF Expression by Human Endothelial Cells in Vitro. In an in vitro angiogenesis assay, IL-25 induced lengthening and branching of microvascular tubules by HUVEC in a concentration-dependent fashion as compared with VEGF, which was used as a positive control (Fig. $3 A-H$ ). IL-25 also induced proliferation and expression of VEGF mRNA by HUVEC (Fig. $3 I$ and $K$ ) and human pulmonary microvascular endothelial cells (HPMEC) (Fig. $3 J$ and $L$ ). These effects were not inhibited by blockade of the IL-17A receptor (IL-17RA) (Fig. $3 I-L)$.

IL-25 Up-Regulated Expression of VEGF Receptors in HUVEC in Vitro. IL-25 also significantly up-regulated expression of mRNA encoding VEGF receptors 1 and 2 (VEGFR1 and VEGFR2) in HUVEC in a time- and concentration-dependent fashion (Fig. 4 $A$ and $B)$. Expression of VEGFR1 and -2 proteins was correspondingly increased with a peak at $\approx 48 \mathrm{~h}$ (Fig. $4 C, D$, and $E$ ).

Effects of Signaling Inhibitors on Angiogenesis and VEGF Protein Production. The PI3K inhibitor LY 294002 and the MEK inhibitor U0126, but not the p38 MAPK inhibitor SB 203580, completely attenuated IL-25-induced formation of new microvascular structures by HUVEC in vitro (Fig. $5 A-C$ ). A similar picture was observed with VEGF-induced angiogenesis. Cellular viability remained $>95 \%$ throughout all cultures. Anti-VEGF neutralizing monoclonal antibody attenuated the angiogenetic effect of IL-25 by up to $70 \%$ and also reduced spontaneous angiogenesis but did not completely ablate the effects of IL-25. Preneutralization of IL-25 with anti-IL-25 antibody completely blocked IL-25-induced angiogenesis. Furthermore, ELISA showed that anti-IL-25 ablated IL-25-induced VEGF production in the angiogenesis assay, and LY294002 and U0126 further reduced it below baseline (Fig. $5 D$ ).
Effects of Signaling Inhibitors on Proliferation and VEGF mRNA Expression by HUVEC. HUVEC were coincubated with IL-25 and a range of signaling inhibitors for $24 \mathrm{~h}$ for quantitative PCR and for $48 \mathrm{~h}$ for the proliferation assay. The proliferation assay
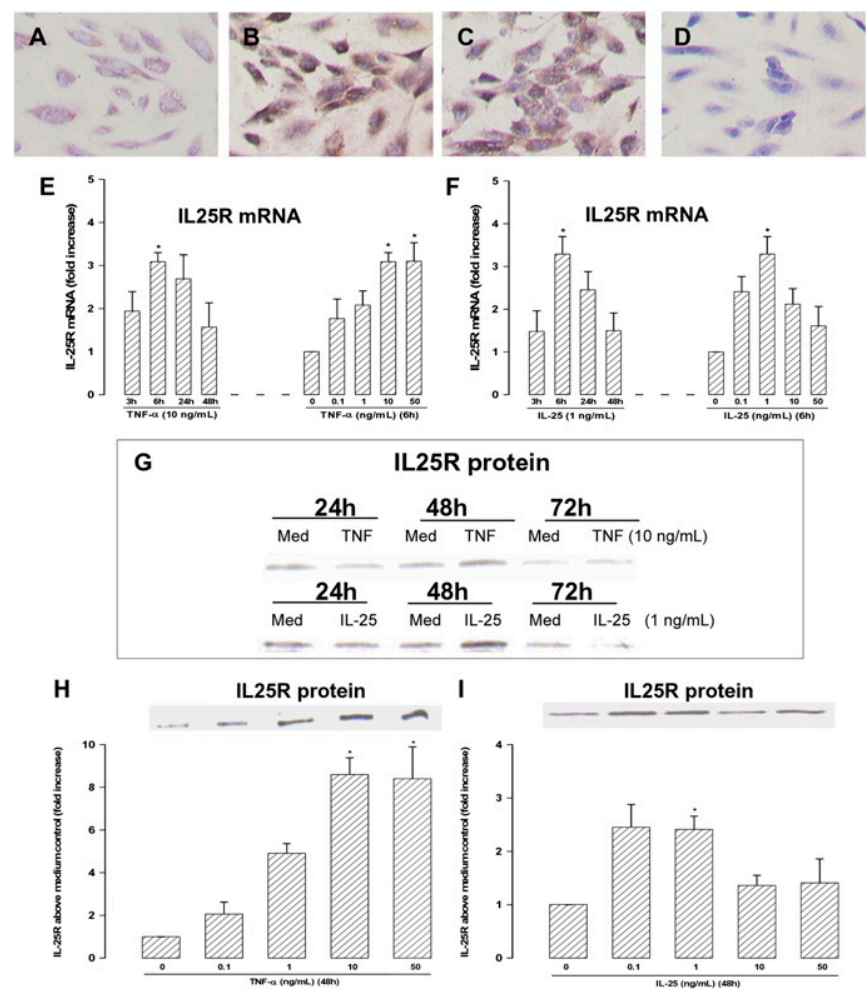

Fig. 2. (A-D) Immunoreactive IL-25R on chamber-cultured HUVEC following culture for $48 \mathrm{~h}$ in $(A)$ medium alone; $(B)$ TNF- $\alpha(10 \mathrm{ng} / \mathrm{mL}) ;(C) \mathrm{IL}-25$ (10 ng/ $\mathrm{mL})$; and $(D)$ as in $B$ but with substitution of the anti-IL-25R antibody with an isotype control (40x magnification). ( $E$ and $F$ ) Real-time PCR showing time courses and concentration response relationships of $(E)$ TNF- $\alpha-$ and $(F)$ IL-25induced IL-25R mRNA expression. (G-I) Western blots showing (G) time course of TNF- $\alpha-(10 \mathrm{ng} / \mathrm{mL})$ and IL-25- $(1 \mathrm{ng} / \mathrm{mL})$ induced IL-25R protein expression, $(H)$ concentration-dependent TNF- $\alpha$-induced, and (I) IL-25induced IL-25R expression at $48 \mathrm{~h}$. Bars represent mean $\pm \operatorname{SEM}(n=4)$. ${ }^{\star} P<$ 0.05 . Mann-Whitney $U$ test. 
Medium

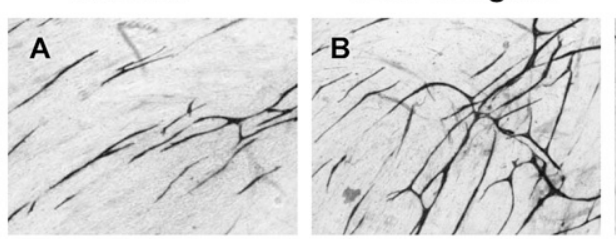

IL-25 $0.1 \mathrm{ng} / \mathrm{mL}$

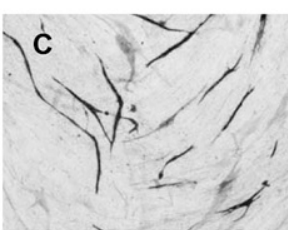

G

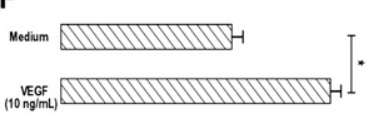

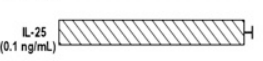

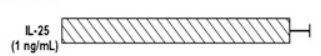

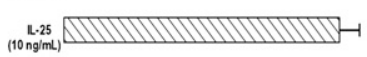

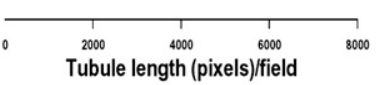

I

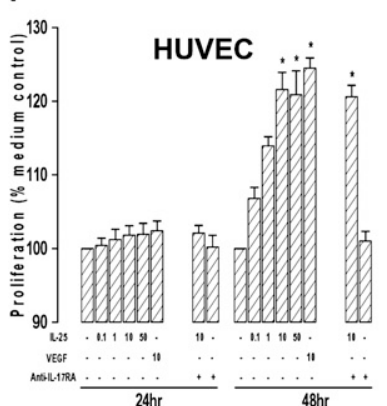

J

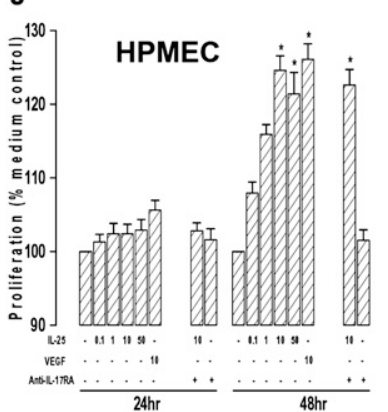

IL-25 $1 \mathrm{ng} / \mathrm{mL}$

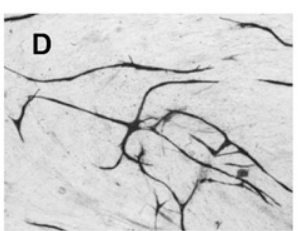

IL-25 $10 \mathrm{ng} / \mathrm{mL}$

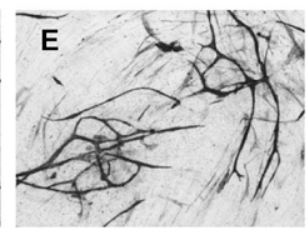

$G$

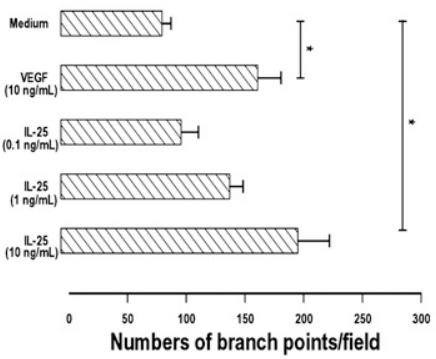

K

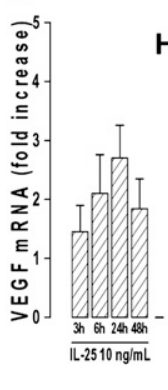

HUVEC

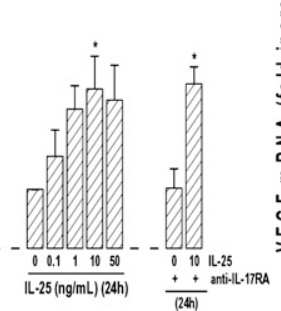

H

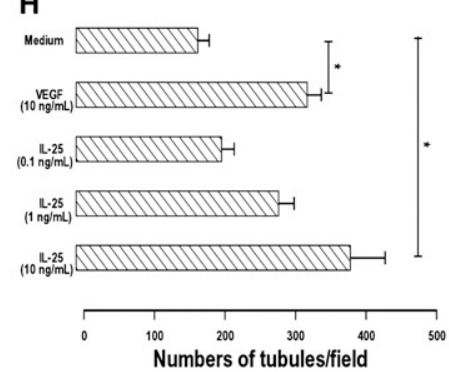

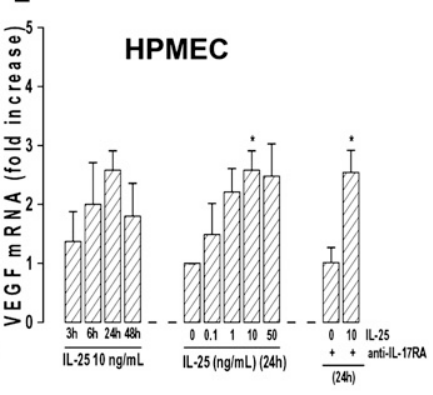

Fig. 3. ( $A-E)$ Representative light photomicrographs (4x original magnification) show formation of primitive vascular tubule structures by HUVEC after $11 \mathrm{~d}$ in culture with medium $(A), \operatorname{VEGF}(10 \mathrm{ng} / \mathrm{mL})(B)$, or IL-25 $[0.1 \mathrm{ng} / \mathrm{mL}(C), 1 \mathrm{ng} / \mathrm{mL}(D)$; or $10 \mathrm{ng} / \mathrm{mL}(E)]$. $(F-H)$ Computer-assisted quantification of numbers $(F)$, branch points $(G)$, and total tubule lengths $(H)$. $(I-L)$ IL-25-induced proliferation of HUVEC $(I)$ and HPMEC $(J)$ and VEGF mRNA expression by HUVEC $(K)$ and HPEMC $(L)$ in the presence or absence of antibody against IL-17RA. Bars show mean \pm SEM of four $(F-H)$ or three $(I-L)$ separate experiments performed in duplicate. ${ }^{*} P<0.05$. Mann-Whitney $U$ test.

showed that the PI3K inhibitor LY294002, the Akt inhibitor SH6, the MEK1/2 inhibitor U0126, the MAPK/ERK1 (MEK1) inhibitor PD98059, and the NF-kB inhibitor Bay11-7082 all significantly attenuated IL-25-induced HUVEC proliferation (Fig. $\mathrm{S} 1 A$ ). In contrast, the JNK inhibitor SP600125, the p38 inhibitor SB203580, and the signal transducer and activator of transcription 3 (Stat3) inhibitor WP-1066 did not show such effects. A similar profile of inhibitory effects was observed on IL-25-induced VEGF mRNA expression in HUVEC by real-time PCR analysis (Fig. $\mathrm{S} 1 B$ )

IL-25-Induced Phosphorylation of Signaling Factors in HUVEC. Western blotting showed that IL-25 activation of HUVEC was accompanied by rapid phosphorylation of PI3K, Akt, ERK1/2, and Stat6 (Fig. S1C). This phosphorylation was evident in some cases as early as at 10 min after IL-25 exposure. IL-25 also induced nuclear translocation of phosphorylated NF- $\kappa$ B (Fig. S1C). In contrast, IL-25 did not affect phosphorylation of Stat3 (Fig. S1C).

\section{Discussion}

We present data consistent with the hypothesis that IL-25, expressed to excess in the asthmatic bronchial mucosa, acts on local vascular endothelial cells, which express its receptor, to stimulate angiogenesis at least partly by inducing elevated expression of VEGF and VEGF receptors.

Our data advance our previous preliminary data (14) showing local elevated expression of IL-25 and IL-25R mRNA in asth- matics. We now show that IL-25 expression increases with disease severity, suggesting the possibility of a functional link. Known cellular sources of IL-25 include activated mast cells, eosinophils, and basophils in humans (13-15) and alveolar macrophages in rats (21). A variety of exogenous stimuli have been postulated to increase IL25 expression (13-15, 22). Importantly, we also discovered elevated numbers of $\mathrm{CD} 31^{+}$vascular endothelial cells expressing IL-25R in the bronchial mucosa of asthmatics.

Functionally, we found that primary cultured human endothelial cells express IL-25R ex vivo and that this expression is increased further by exposure to nanomolar quantities of IL-25 itself, suggesting that IL-25 increases the sensitivity of vascular endothelial cells to its own effects. To check the generality of these observations, we compared the effects of IL-25 on human endothelial cells of umbilical vein and pulmonary origin and found essentially identical effects (Fig. 3). We used TNF- $\alpha$ as a tentative positive control for these experiments because this cytokine previously had been shown to up-regulate IL-25R expression on human lung fibroblasts and smooth muscle cells (17, 19). That TNF- $\alpha$ did increase IL-25R expression on endothelial cells suggests a possible mechanism linking $\mathrm{TNF}-\alpha$, already clearly implicated in asthma pathogenesis, with remodeling changes. Furthermore, although the potential sources of TNF- $\alpha$ in asthma are legion, endothelial cells may contribute (23). In addition to increasing IL-25R expression, IL-25 enhanced HUVEC proliferation and assembly into branching microvessels reminiscent of capillary networks in vivo. As a positive control, we compared the effects of VEGF with those of IL-25 and found 
A
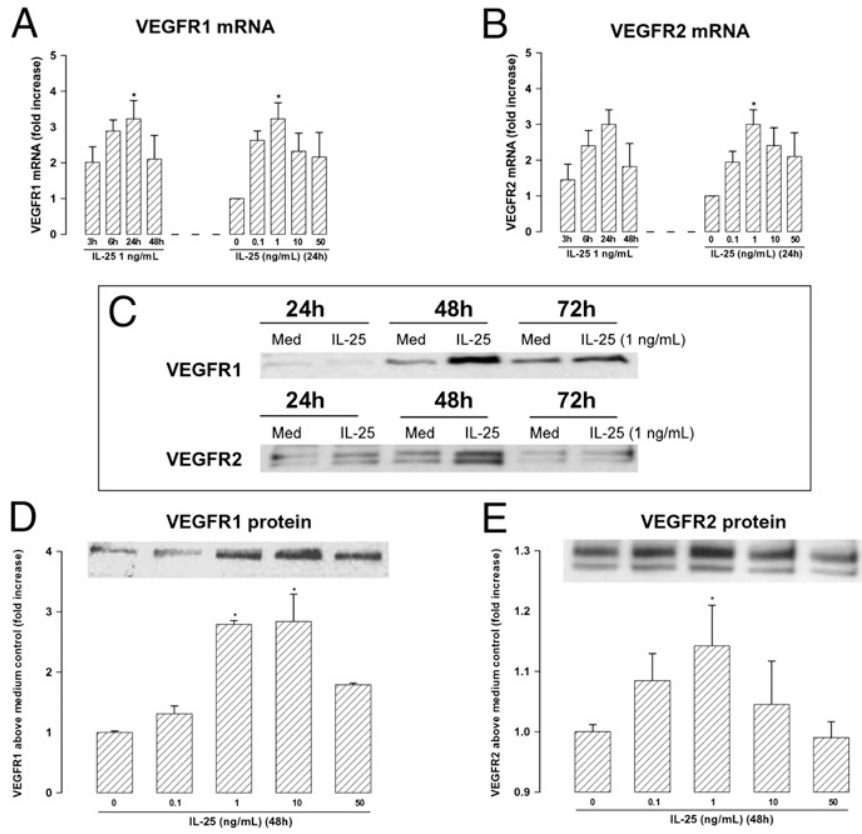

Fig. 4. ( $A$ and $B$ ) Real-time $P C R$ amplification of mRNA encoding $(A)$ VEGFR1 and $(B)$ VEGFR2 in HUVEC stimulated with IL-25. (C-E) Western blots of (C) the peak expression of VEGFR1 and -2 proteins and concentration-dependent induction of (D) VEGFR1 and (E) VEGFR2 by IL-25 at $48 \mathrm{~h}$ in cultured HUVEC. Bars show mean $\pm \operatorname{SEM}(n=4)$. ${ }^{*} P<0.05$. Mann-Whitney $U$ test.

them to be comparable. Spontaneous and IL-25-induced angiogenesis was extensively but not completely inhibited by VEGF blockade, suggesting that IL-25 acts principally by increasing
VEGF production. This incomplete inhibition was verified by VEGF ELISA, although a possible direct effect of IL-25 on endothelial cells or the possibility that IL-25 induces the production of angiogenetic mediators other than VEGF cannot be excluded. We then verified that IL-25 increases the expression not only of VEGF but also its two principal receptors, VEGFR1 and -2 , in vascular endothelial cells, again suggesting that IL-25 increases the potential capacity of these cells to respond to VEGF. The bell-shaped concentration-response curves for IL25 in inducing expression of IL-25R, VEGF, and VEGF receptors by HUVEC (Figs. 2, 3, and 4) are compatible with the hypothesis that higher concentrations of IL-25 induce IL-25R internalization, as observed with some cytokines and chemokines and their receptors (e.g., as IL-5 and eotaxin) $(24,25)$.

Recent reports $(26,27)$ have suggested that endothelial cells also express IL-17RA and that certain activities of IL-25 can be exerted through this receptor. Therefore we examined the effects of an anti-IL-17RA blocking antibody. Anti-IL-17RA did not attenuate endothelial cell proliferation or VEGF expression (Fig. 3).

The signaling pathways used by IL- 25 when acting on its receptor IL-25R have not been well defined and may vary by cell type and species. Previous studies $(16,28)$ suggest a role for p38 MAPK, $\mathrm{JNK}$, and NF- $\mathrm{KB}$ in T cells and eosinophils in humans and TNF receptor-associated factor 6 and the adaptor protein connection to IK K-complex and SAPK (CIKS)/Act in mice (29-31). To investigate the signaling pathways involved in IL-25-induced VEGF expression, we used a panel of commercially available inhibitors. Inhibition of PI3K or MEK1/2 with the pharmacological inhibitors LY294002/SH-6 and U0126, respectively, completely attenuated IL-25-induced VEGF mRNA expression and proliferation in endothelial cells. Inhibition of Akt (SH-6), MEK1 alone (with PD98059), or NF-kB (with Bay 11-7082) showed measurable but lesser effects, whereas the Stat3 inhibitor WP-1066, the P38 MAPK inhibitor SB203580, and the JNK inhibitor SP600125 were ineffective. Taking together these and previously published data, we
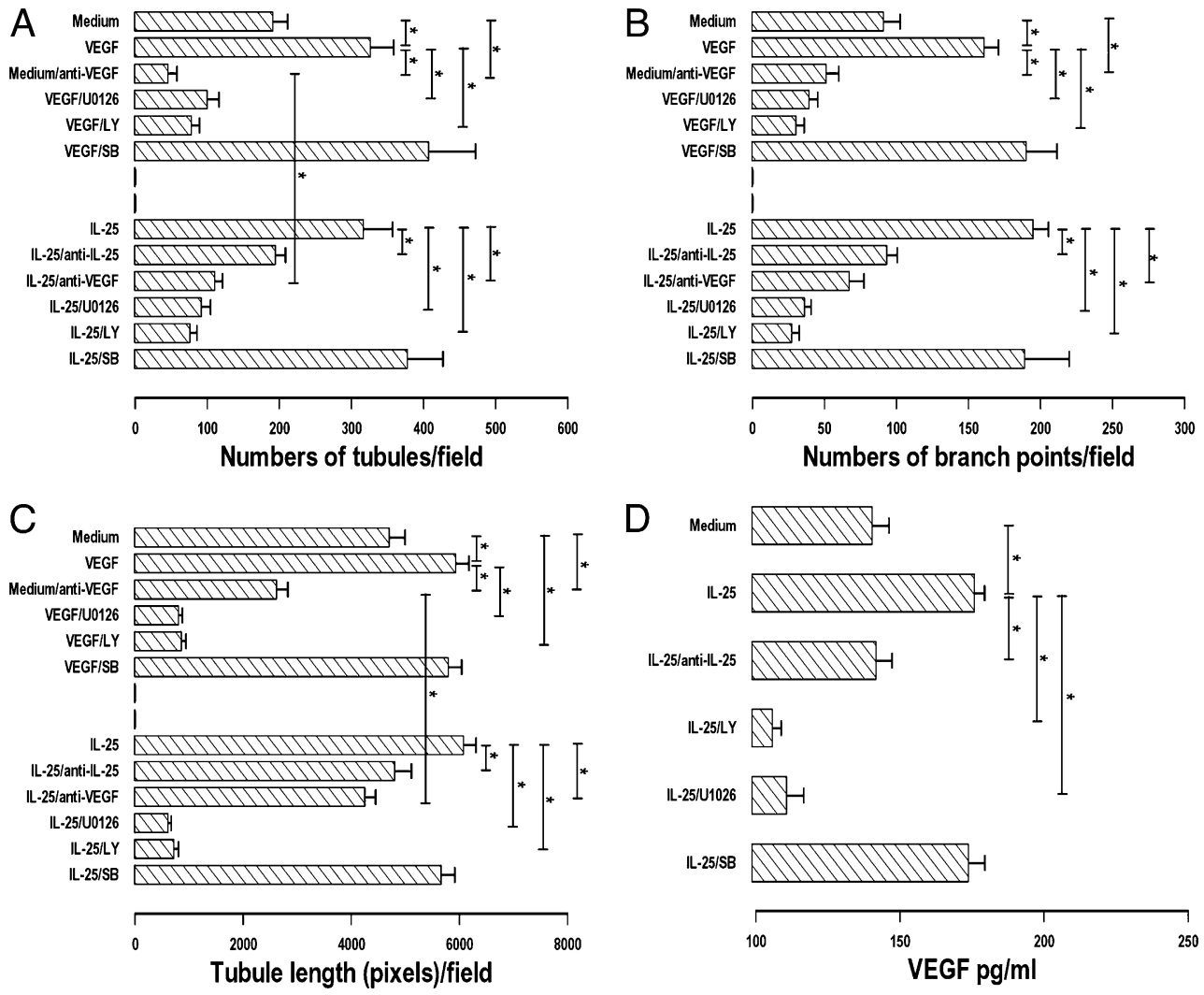

Fig. 5. (A-C) Effects of anti-VEGF $(1 \mu \mathrm{g} / \mathrm{mL})$, anti-IL-25 $(1 \mu \mathrm{g} / \mathrm{mL})$, the PI3K inhibitor LY29404, the MEK1/2 inhibitor U0126, and the P38MAPK inhibitor SB203580 $(10 \mu \mathrm{M} / \mathrm{L})$ on VEGF- and IL-25induced angiogenesis in 11-d cultures. (A) Number of tubules per field. $(B)$ Number of branch points per field. (C) Tubule length (in pixels) per field. Bars show the mean \pm SEM of four separate experiments performed in duplicate. $(D)$ ELISA measurement of VEGF protein in the supernatants of IL-25-induced angiogenesis assays (day 9 of culture). $* P<$ 0.05. Mann-Whitney $U$ test. 
have summarized in Fig. S2 the signaling pathways that, to date, have and have not been implicated in IL-25 signaling. For reasons of practicality and cost, we did not perform full concentrationresponse curves with all the signaling inhibitors but used concentrations that were physiologically relevant but did not compromise the viability of the endothelial cells. The effects of these inhibitors on VEGF production and VEGF-induced vascular formation in the present study closely mirrored their activities in inhibiting IL25-enhanced angiogenesis. These data are consistent with a role for PI3K/Akt and MEK/Erk signaling in IL-25-induced angiogenesis, although they do not allow discrimination between effects on IL-25 signaling inducing VEGF and VEGFR expression or VEGF signaling inducing angiogenesis, or both. Our data suggest that blocking PI3K, MEK, and NF- $\mathrm{KB}$ reduces IL-25-induced proliferation and VEGF expression by endothelial cells, two critical factors in the regulation of angiogenesis.

Other recent studies also suggest that PI3K plays a critical role in cytokine production and angiogenesis by vascular endothelial cells (32-34); indeed, it has been suggested more generally that PI3K may be a key molecular target for the therapy of asthma (35). In the present study, we were unable to examine inhibition of Stat6 signaling because of the lack of commercially available specific inhibitors. However, we did observe that IL-25 enhanced phosphorylation of signal transducer and activator of transcription 6 (Stat6) but not of Stat3, suggesting that Stat6 also might mediate IL-25-induced angiogenesis through its effects on the proliferation of endothelial cells and expression of VEGF. Some caution must be exercised when interpreting our data showing an apparent lack of effect of the p38 MAPK inhibitor SB203580 and the JNK inhibitor SP600125 on IL-25-induced VEGF mRNA expression and angiogenesis, because, as mentioned, one may be observing the net effect of influences on IL-25- and VEGF-mediated signaling. Issbrücker and colleagues (36) previously reported that SB203580 enhanced VEGF-induced angiogenesis by endothelial cells.

Increased angiogenesis is a well-documented feature of airways remodeling in asthma. We (37) and others (38) have shown elevated expression of angiogenetic mediators, including VEGF, fibroblast growth factor, platelet-derived growth factor, and angiogenin in the airways tissue and lining fluid of asthmatics, and the expression of these mediators can be correlated positively with disease severity and negatively with airways obstruction. VEGF appears to be key among these mediators, and IL-25 may make a significant contribution to its expression. Because airways fibroblasts and smooth muscle cells also express IL-25R $(17,19)$, we speculate that IL-25 may play an even wider role in airways remodeling through its effects on fibrogenesis and smooth muscle hyperplasia. For the moment, our data extend the knowledge of the manifold possible roles for IL25 in asthma pathogenesis and point to it and its putative signaling molecules PI3K/Akt and MEK1/2/Erk as candidate molecular targets for novel therapeutic approaches.

\section{Methods}

Study Patients. The study was approved by the Guy's Hospital Ethics Committee and was carried out in our clinical research facility. Each subject provided written, informed consent. Endobronchial biopsies were obtained from 15 asthmatics and 15 normal controls. Details are given in SI Methods and Table S1.

Fiberoptic Bronchoscopy. Fiberoptic bronchoscopy was performed as previously described $(39,40)$. Details are given in SI Methods.

Immunohistochemistry. To identify cells expressing IL-25, IL-25R, and CD31, we used the primary antibodies mouse anti-human IL-17E, MAB 1258 (1:20; R\&D Systems), mouse anti-human IL-25R (1:50; LB-2044; Autogene Bioscience), and mouse anti-human CD31 (1:30; M0823; Dako Ltd.) and well-established protocols that we have described previously $(39,40)$. Positively staining cells were detected with Fast Red (Sigma) $(39,40)$. Further details are given in SI Methods The mean \pm SD of the entire cross-sectional areas of the biopsy sections ex amined in the asthma and control groups were $2.6 \pm 0.3 \mathrm{~mm}^{2}$ and $2.8 \pm 0.4 \mathrm{~mm}^{2}$ respectively, with within-group coefficients of variation ranging from 8.79 $10.2 \%$. The between-observer coefficients of variation from duplicate counts for all markers tested for two groups ranged from 3.2-5.1\%.
HUVEC Culture. All reagents and HUVEC were purchased from PromoCell unless otherwise specified. Cells before passage four were used for experiments. Briefly, cells $\left(0.5 \times 10^{6}\right.$ per well) were seeded in six-well plates (Nunc $\mathrm{A} / \mathrm{S}$ ) precoated with gelatin (0.5\%; Sigma-Aldrich) in complete medium (endothelial cell media kit; PromoCell) containing basal medium, $0.4 \%$ endothelial cell growth supplement, $2 \% \mathrm{FCS}, 0.1 \mathrm{ng} / \mathrm{mL}$ EGF, $1 \mathrm{ng} / \mathrm{mL}$ basic FGF, $100 \mathrm{U} / \mathrm{mL}$ penicillin, and $100 \mu \mathrm{g} / \mathrm{mL}$ streptomycin and grown to $\approx 80 \%$ confluence in a $5 \% \mathrm{CO}_{2}$ incubator at $37{ }^{\circ} \mathrm{C}$. The cells then were exposed to stimuli including recombinant human TNF- $\alpha$ (R\&D) and IL-25 (R\&D Systems). To identify IL-25R immunoreactivity on HUVEC, some HUVEC were seeded and cultured to $\approx 80 \%$ confluence in chamber slides (Nalge Nunc International) for $48 \mathrm{~h}$ with or without recombinant human TNF- $\alpha$. The effects of a number of signaling inhibitors were tested in this system; a full description is given in SI Methods. Cells were harvested for further investigation at different time points, as described in Results. Cellular viability was assessed by Trypan blue exclusion.

RNA Extraction and Real-Time PCR. Total cellular RNA was extracted from HUVEC using the RNeasy Mini kit (QIAGEN). The RNA was reverse transcribed and subjected to quantitative real-time PCR analysis (further details are given in SI Methods). Real-time PCR primers were 5'-AACAGGCGTCCCTTTCCCTCTGGA-3' and 5'-TTCTTGATCCTTTCGTGCCTCCAC-3' for human IL-25R (IL17BR); 5' - TACCTCCACCATGCCAAGTG-3' and 5'-GATGATTCTGCCCTCCTCCTT3' for human VEGF; 5'-TCACTGCCACTCTAATTGTCAATGT-3' and 5'-AAACGATGACACGGCCTTTT-3' for human VEGF receptor 1 (VEGFR1), and 5'CAAGACAGGAAGACCAAGAAAAGAC-3' and 5'-GGTGCCACACGCTCTAGGA$3^{\prime}$ for human VEGF receptor 2 (VEGFR2.).

Western Blotting. Western blotting was performed as previously described (38). HUVEC were cultured with or without stimuli for 24,48 , or $72 \mathrm{~h}$ in a $5 \% \mathrm{CO}_{2}$ incubator at $37{ }^{\circ} \mathrm{C}$. Cells were washed with ice-cold PBS and then lysed in $50 \mu \mathrm{L}$ lysis buffer (25 mM Hepes, 1.5\% Triton X-100, 0.1\% SDS, $0.5 \mathrm{M} \mathrm{NaCl}, 5 \mathrm{mM}$ EDTA, $0.1 \mathrm{mM}$ sodium deoxycholate; Sigma) containing a protease inhibitor mixture (Roche) and $10 \mathrm{mM}$ sodium orthovanadate (Sigma). Further details are given in SI Methods. The receptor proteins were detected by incubation with antibodies against human IL-25R (1:2,000; LifeSpan Biosciences) (17), VEGFR1 (1:4,000; Abcam plc) and VEGFR2 (1:1,000; Cell Signaling Technology, Inc.) (41) for 16 h. After washing, the membrane was incubated with HRP-conjugated goat antimouse for IL-25R detection (1:3,000; Dako) or HRP-conjugated goat antirabbit for VEGFR detection (1:10,000; Abcam) in blocking buffer for 45 $\min$ at room temperature and subsequently were developed with Western blotting detection reagents (Amersham) according to the manufacturer's instructions.

In separate experiments, cells were incubated with or without IL-25 (R\&D Systems) to detect IL-25-induced phosphorylation of signaling molecules (42). Antibodies were purchased from Cell Signaling Technology, Inc. and are listed, along with the dilutions used, in SI Methods. Total GAPDH and nuclear matrix protein p84 were detected by monoclonal antibodies (GAPDH: WH0002597M1; 1:5,000; Sigma; nuclear matrix protein p84: ab487; 1:1,000; Abcam plc) as loading controls for cytoplasmic and nuclear proteins, respectively $(43,44)$

HUVEC Proliferation Assay. HUVEC proliferation was measured using a commercial kit (WST-8, Cayman Chemicals) (45). Briefly, $1 \times 10^{4}$ cells in $100 \mu \mathrm{L}$ of medium were plated on a 96-well microplate. After $24 \mathrm{~h}$ of incubation, aliquots of $100 \mu \mathrm{L}$ medium with several concentrations of recombinant human IL25 were added. After incubation for 24 or $48 \mathrm{~h}$, the medium was replaced with new medium containing $10 \%$ WST- 8 . After $1 \mathrm{~h}$ of incubation, the formation of formazan was determined photometrically at $450 \mathrm{~nm}$ with a microplate reader. Recombinant human VEGF $(10 \mathrm{ng} / \mathrm{mL})$ was used as a positive control. All analyses were performed on sets of four wells. The results are expressed as percentages of medium control values. To explore the generality of these properties of endothelial cells, similar experiments were performed on microvascular endothelial cells of pulmonary origin (PromoCell $\mathrm{GmbH}$ ). To investigate whether IL-25 binds to the alternative receptor IL-17RA $(26,27)$, cells were precultured with $4 \mu \mathrm{g} / \mathrm{mL}$ anti-IL-17RA monoclonal neutralizing antibody (MAB177; R\&D Systems) for 30 min before recombinant human IL-25 (10 ng/ $\mathrm{mL}$ ) was added for a further 24-h incubation. After culturing, cells were harvested for proliferation assays and real-time PCR to measure VEGF mRNA expression, as described.

In Vitro Angiogenesis Assay. The effects of IL-25 on angiogenesis were investigated using an in vitro angiogenesis assay $(34,35)$ based on coculture of HUVEC for $11 \mathrm{~d}$ over a monolayer of irradiated fibroblasts (AngioKit; TCS 
CellWorks). Further details are given in SI Methods. To evaluate whether VEGF is involved in IL-25-induced angiogenesis, a monoclonal neutralizing antibody against human VEGF ( $1 \mu \mathrm{g} / \mathrm{mL}$ ) (R\&D Systems) was added to some wells on day 0 and replenished on days 4, 7, and 9. Preincubation of IL-25 with anti-IL-25 antibody ( $1 \mu \mathrm{g} / \mathrm{mL}$ ) (R\&D Systems) also was used to neutralize IL-25. Additionally, IL-25 signaling pathways inducing angiogenesis were probed by adding specific inhibitors including SB203580, U0126, and LY294002 $(10.0 \mu \mathrm{mol} / \mathrm{L}$ for the first $3 \mathrm{~d}$, followed by $2.0 \mu \mathrm{mol} / \mathrm{L}$ until the end of the 11-d culture period) $(31,34)$.

ELISA for VEGF. At day 9, supernatants were collected from the angiogenesis assays. ELISA kits were used to measure human VEGF according to the manufacturer's instructions (R\&D Systems). The limit of detection was $63 \mathrm{pg} / \mathrm{mL}$.

1. Kristan SS, et al. (2009) Airway angiogenesis in patients with rhinitis and controlled asthma. Clin Exp Allergy 39:354-360.

2. Li X, Wilson JW (1997) Increased vascularity of the bronchial mucosa in mild asthma. Am J Respir Crit Care Med 156:229-233.

3. Hoshino M, Takahashi M, Aoike N (2001) Expression of vascular endothelial growth factor, basic fibroblast growth factor, and angiogenin immunoreactivity in asthmatic airways and its relationship to angiogenesis. J Allergy Clin Immunol 107:295-301.

4. McDonald DM (2001) Angiogenesis and remodelling of airway vasculature in chronic inflammation. Am J Respir Crit Care Med 164:39-45.

5. Puxeddu I, et al. (2009) The role of eosinophil major basic protein in angiogenesis. Allergy 64:368-374.

6. Detoraki A, et al. (2010) Angiogenesis and lymphangiogenesis in bronchial asthma. Allergy 65:946-958.

7. Dong C (2008) Regulation and pro-inflammatory function of interleukin-17 family cytokines. Immunol Rev 226:80-86.

8. Fort MM, et al. (2001) IL-25 induces IL-4, IL-5, and IL-13 and Th2-associated pathologies in vivo. Immunity 15:985-995.

9. Hurst SD, et al. (2002) New IL-17 family members promote Th1 or Th2 responses in the lung: In vivo function of the novel cytokine IL-25. J Immunol 169:443-453.

10. Pan G, et al. (2001) Forced expression of murine IL-17E induces growth retardation, jaundice, a Th2-biased response, and multiorgan inflammation in mice. $J$ Immunol 167:6559-6567.

11. Kim MR, et al. (2002) Transgenic overexpression of human IL-17E results in eosinophilia, B-lymphocyte hyperplasia, and altered antibody production. Blood 100: 2330-2340.

12. Ballantyne SJ, et al. (2007) Blocking IL-25 prevents airway hyperresponsiveness in allergic asthma. J Allergy Clin Immunol 120:1324-1331.

13. Angkasekwinai $P$, et al. (2007) Interleukin 25 promotes the initiation of proallergic type 2 responses. J Exp Med 204:1509-1517.

14. Wang YH, et al. (2007) IL-25 augments type 2 immune responses by enhancing the expansion and functions of TSLP-DC-activated Th2 memory cells. J Exp Med 204: 1837-1847.

15. Ikeda K, et al. (2003) Mast cells produce interleukin-25 upon Fc epsilon RI-mediated activation. Blood 101:3594-3596.

16. Wong CK, Cheung PF, Ip WK, Lam CW (2005) Interleukin-25-induced chemokines and interleukin- 6 release from eosinophils is mediated by $\mathrm{p} 38$ mitogen-activated protein kinase, c-Jun N-terminal kinase, and nuclear factor-kappaB. Am J Respir Cell Mol Biol 33:186-194.

17. Lajoie-Kadoch $\mathrm{S}$, et al. (2006) TNF-alpha and IFN-gamma inversely modulate expression of the IL-17E receptor in airway smooth muscle cells. Am J Physiol Lung Cell Mol Physiol 290:L1238-L1246.

18. Caruso R, et al. (2009) Inhibition of monocyte-derived inflammatory cytokines by IL-25 occurs via p38 Map kinase-dependent induction of Socs-3. Blood 113:3512-3519.

19. Létuvé S, et al. (2006) IL-17E upregulates the expression of proinflammatory cytokines in lung fibroblasts. J Allergy Clin Immunol 117:590-596.

20. Meng Q, et al. (2009) IL-25 and its receptor (IL-25R) in allergic inflammation: Its role in VEGF-mediated angiogenesis. J Allergy Clin Immunol 123:S58 [abs 209].

21. Kang CM, et al. (2005) Interleukin-25 and interleukin-13 production by alveolar macrophages in response to particles. Am J Respir Cell Mol Biol 33:290-296.

22. Wang YH, Liu YJ (2009) Thymic stromal lymphopoietin, OX40-ligand, and interleukin25 in allergic responses. Clin Exp Allergy 39:798-806.

23. Imaizumi T, et al. (2000) Expression of tumor necrosis factor-alpha in cultured human endothelial cells stimulated with lipopolysaccharide or interleukin-1alpha. Arterioscler Thromb Vasc Biol 20:410-415.
Statistical Analysis. Data were analyzed using the Minitab Release 7statistical package (Minitab Inc.). The Mann-Whitney $U$ test was used for analyzing all data, and $P<0.05$ was considered significant.

ACKNOWLEDGMENTS. The authors are grateful to Kheem Jones, Cherylin Reinholtz, Karen Williams, Helen Bull, and Drs. Ghada Eid and Leonard Siew in the Department of Asthma, Allergy and Respiratory Science, King's College London, for their help in recruiting patients, collecting endobronchial biopsies, and documenting clinical information. This work was supported by the Dana Foundation (Y.-J.L. and S.Y.), Asthma UK (S.Y.), and the Friends of Guy's Hospital, London (S.Y.). Financial support also was provided by the Department of Health via a National Institute for Health Research comprehensive Biomedical Research Centre award to Guy's and St Thomas' National Health Service Foundation Trust in partnership with King's College London and King's College Hospital National Health Service Foundation Trust.

24. Lei JT, Martinez-Moczygemba M (2008) Separate endocytic pathways regulate IL-5 receptor internalization and signaling. J Leukoc Biol 84:499-509.

25. Zimmermann N, Conkright JJ, Rothenberg ME (1999) CC chemokine receptor-3 undergoes prolonged ligand-induced internalization. J Biol Chem 274:12611-12618.

26. Roussel L, et al. (2010) IL-17 promotes p38 MAPK-dependent endothelial activation enhancing neutrophil recruitment to sites of inflammation. $J$ Immunol 184:4531-4537.

27. Rickel EA, et al. (2008) Identification of functional roles for both IL-17RB and IL-17RA in mediating IL-25-induced activities. J Immunol 181:4299-4310.

28. Wong CK, Li PW, Lam CW (2007) Intracellular JNK, p38 MAPK and NF-kappaB regulate IL-25 induced release of cytokines and chemokines from costimulated $\mathrm{T}$ helper lymphocytes. Immunol Lett 112:82-91.

29. Maezawa Y, et al. (2006) Involvement of TNF receptor-associated factor 6 in IL-25 receptor signaling. J Immunol 176:1013-1018.

30. Claudio E, et al. (2009) The adaptor protein CIKS/Act1 is essential for IL-25-mediated allergic airway inflammation. J Immunol 182:1617-1630.

31. Swaidani S, et al. (2009) The critical role of epithelial-derived Act1 in IL-17- and IL-25 mediated pulmonary inflammation. J Immunol 182:1631-1640.

32. Choi YK, et al. (2010) Carbon monoxide promotes VEGF expression by increasing HIF$1 \alpha$ protein level via two distinct mechanisms, translational activation and stabilization of HIF- $1 \alpha$ protein. J Biol Chem 285:32116-32125.

33. Weylie B, Zhu J, Singh U, Ambrus S, Forough R (2006) Phosphatidylinositide 3-kinase is important in late-stage fibroblast growth factor-1-mediated angiogenesis in vivo. $J$ Vasc Res 43:61-69.

34. Wang Z, Jiang C, Ganther H, Lü J (2001) Antimitogenic and proapoptotic activities of methylseleninic acid in vascular endothelial cells and associated effects on PI3K-AKT, ERK, JNK and p38 MAPK signaling. Cancer Res 61:7171-7178.

35. Takeda M, et al. (2010) The pathophysiological roles of PI3Ks and therapeutic potential of selective inhibitors in allergic inflammation. Int Arch Allergy Immunol 152(Suppl 1):90-95.

36. Issbrücker K, et al. (2003) p38 MAP kinase-a molecular switch between VEGFinduced angiogenesis and vascular hyperpermeability. FASEB $J$ 17:262-264.

37. Simcock DE, et al. (2007) Proangiogenic activity in bronchoalveolar lavage fluid from patients with asthma. Am J Respir Crit Care Med 176:146-153.

38. Bailey SR, et al. (2009) Airway vascular reactivity and vascularisation in human chronic airway disease. Pulm Pharmacol Ther doi:10.1016.

39. Ying S, et al. (2005) Thymic stromal lymphopoietin expression is increased in asthmatic airways and correlates with expression of Th2-attracting chemokines and disease severity. J Immunol 174:8183-8190.

40. Ying $S$, et al. (2008) Expression and cellular provenance of thymic stromal lymphopoietin and chemokines in patients with severe asthma and chronic obstructive pulmonary disease. J Immunol 181:2790-2798.

41. Lin CM, et al. (2006) Protective role of wogonin against lipopolysaccharide-induced angiogenesis via VEGFR-2, not VEGFR-1. Int Immunopharmacol 6:1690-1698.

42. Abid MR, et al. (2004) Vascular endothelial growth factor-mediated induction of manganese superoxide dismutase occurs through redox-dependent regulation of forkhead and IкB-NF-кB. J Bio Chem 279:44030-44038.

43. Ye M, et al. (2008) Involvement of PI3K/Akt signaling pathway in hepatocyte growth factor-induced migration of uveal melanoma cells. Invest Ophthalmol Vis Sci 49 497-504.

44. Benko S, Magalhaes JG, Philpott DJ, Girardin SE (2010) NLRC5 limits the activation of inflammatory pathways. J Immunol 185:1681-1691.

45. Fujii T, Wakaizumi M, Ikami T, Saito M (2008) Amla (Emblica officinalis Gaertn-) extract promotes procollagen production and inhibits matrix metalloproteinase- 1 in human skin fibroblasts. J Ethnopharmacol 119:53-57. 